\title{
Ultrafast electron microscopy
}

Reinventing femtosecond atomic-scale imaging

Transmission electron
microscopy may have brought
about some of the most
cutting-edge research into
materials physics, but the
technique doesn't come
without its limitations. In
his research, Dr Jinfeng Yang
at Osaka University in Japan
aims to further improve
the capabilities of the most
powerful microscopes ever
made, by accelerating the
pulsed beams of electrons
they produce to relativistic
speeds. Through several
recent experiments, he and
his team have shown that their
updated technique of 'ultrafast
electron microscopy' can image
atomic-scale processes directly
as they unfold.

\section{hysicists have been measuring} the properties of materials for many centuries. For most of this time, such research has involved variety of ways: from poking and stretching to squeening and crushing. Many useful physical laws have been constructed through measurements of how materials respond in these experiments, but to truly understand what is going on, more sophisticated apparatus would be needed.

In reality, the material properties we observe on macroscopic scales result from the countless interactions which take place between individual atoms, on timescales as fast as quadrilionth of a second - or 'femtoseconds. To study processes on such intricate scales, physicists currently use the technique of timeresolved transmissic

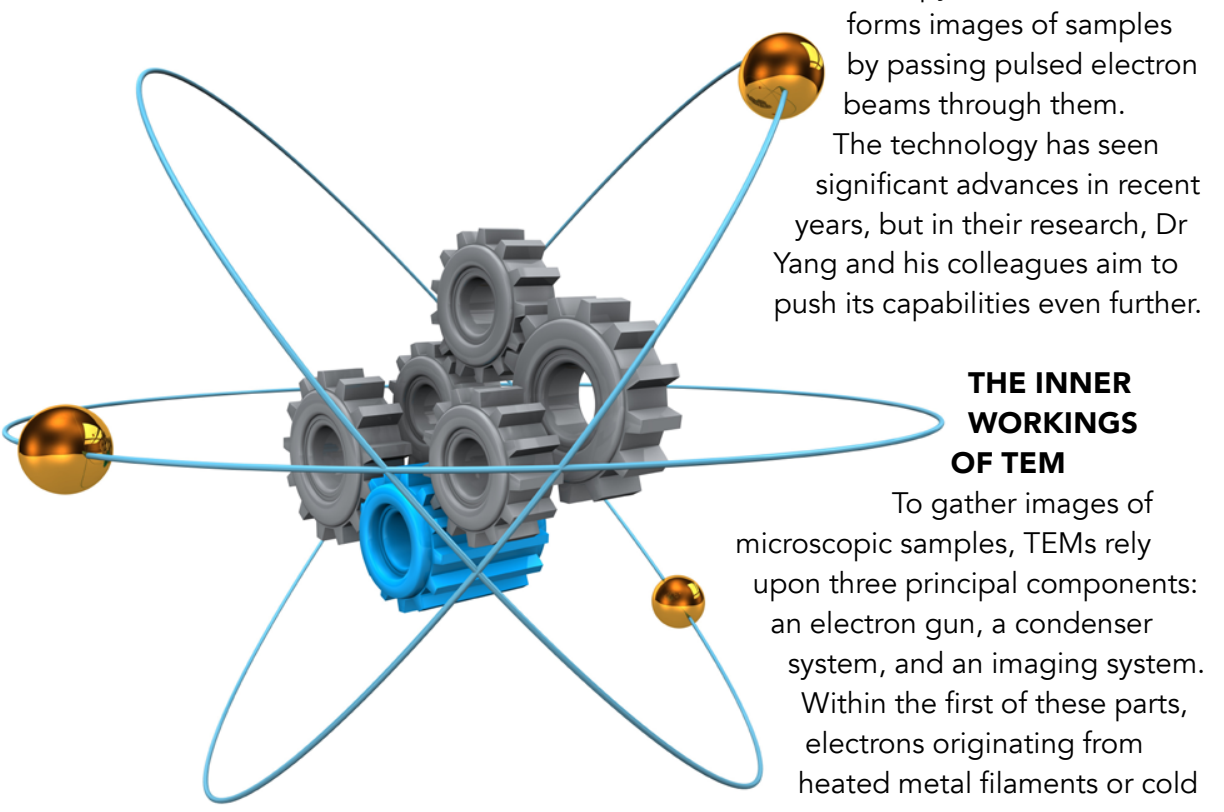

field-emission cathodes are accelerated towards an aperture-shaped electrode by a strong electric field, produced by a direct current. Provided the voltage table, the two elctrods is highly through the hole in the electrode.

completing the 'gun' component, Next, the electrons pass through a series of condenser lenses, which use magnetic fields to precisely control the intensity of the beam, and its illumination angle on the sample.

Atter passing through the sample, the altered electron beam is then magnified many times by a series of objective

lenses, eventually reaching macroscopic scales. Finally, the electrons interact with a device that converts them in photons of visible light, producing a final image of the sample. Since the wavelengths of electrons, as

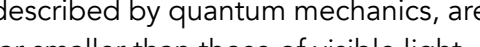
TEM allows for 3D images with far higher resolutions than traditional light microscopes, enabling researchers to directly obtain images of individual toms and molecules.

\section{CHALLENGES WITH FEMTOSECOND}

ATOMIC-SCALE IMAGING
So far, this setup has allowed

researchers to study systems as small as individual atoms, but significant limitations remain. The first major shortcoming with time-resolved TEM is the relatively long duration of the electron beams it produces in singleshot experiments, which are used the shortest possible processes on addition, beam multi-scattering within

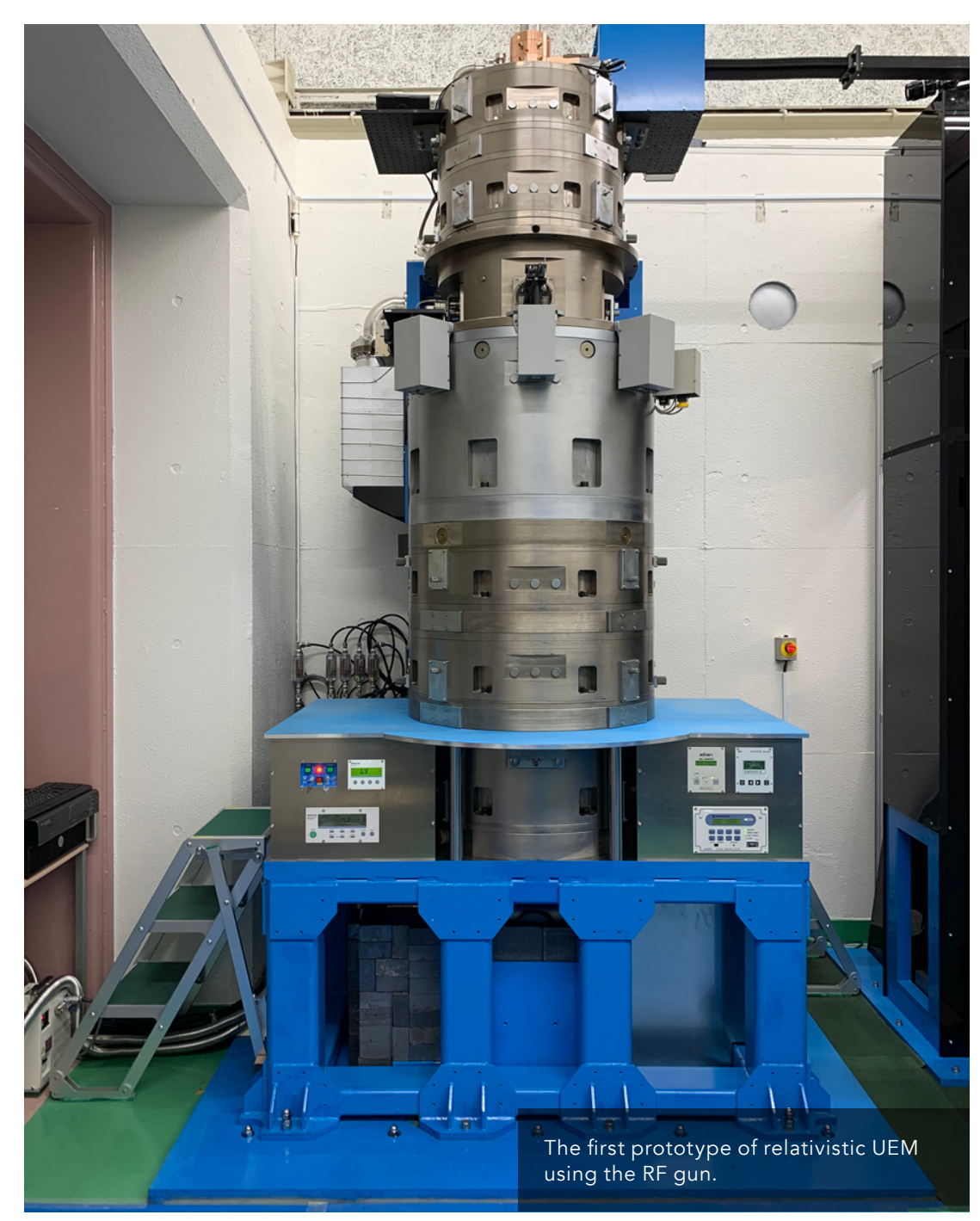

Dr Yang's team observed UEM images with $3 \mathrm{MeV}$ femtosecond electron pulses and succeeded in single-shot imaging at a lowmagnification observation.

samples is currently limiting the spatial research, Dr Yang proposes that each of these issues could be solved throug the use of relativistic electron beams. Unlike the beams used in time-resolved TEM, these electrons would travel at ignificant fractions of the speed of light; so fast that the effects described by Einstein's theory of general relativity become relevant.

"Femtosecond atomic-scale imaging js the most challenging subject in "In our research, "explans Dryang. an innovative, relativistic ultrafast electron microscope (UEM) with an ultimate temporal-spatial resolution angstrom. Then, we aim to realise the real time, and on atomic scales." Since setting out these goals in a 2013 study, Dr Yang's team has made significant realisation of UEM devices.

THE RADIO-FREQUENCY GUN The electron gun was the first el of 10 femtoseconds (fs) and 1 strides towards the widespread the component is limited because of its use of direct current, which as the low energies of up to 200 kiloelectronvolts (keV). They can produce emtosecond electron pulses, but the pulses only have relatively low electron numbers per pulse - typically maximum of several thousandwhich is not large enough for the single-shot experiments required on femtosecond timescales.

To solve this problem, Dr Yang and his colleagues have developed an updated electron gun, which accelerates the electrons using cilio frequencies instead of direct current. This approach subjects beams trongr, meaning they can be quick accelerad to the relativistic quickly required for UEM. "A radio-frequency (RF) acceleration-based electron gun has been developed to successfully generate a high-current femtosecondpulsed electron beam with an energy of $3 \mathrm{MeV}$, and a pulse duration of 100 femtoseconds," Dr Yang summarises. With this technological breakthrough the researchers were then able to move on to realising the practical side

\section{CREATING IMAGES WITH UEM} To achieve actual images of atomicscale structures on ultrafast timescal the researchers have developed in electron gun can better concentrate the electron beam onto a sample. "Using the RF gun, we have developed the world's first prototype of relativistic UEM," Dr Yang describes. "We succeeded in observing UEM images of gold nanoparticles and polystyrene particles with $3 \mathrm{MeV}$ femtosecond electron pulses. At a low-magnification observation, like in the observation of microcrystals, single-shot imaging with the relativistic femtosecond electron pulse is achievable."

With such advanced capabilities of single-shot imaging, the researchers to directly in the irre be processes in magerias as they transito 


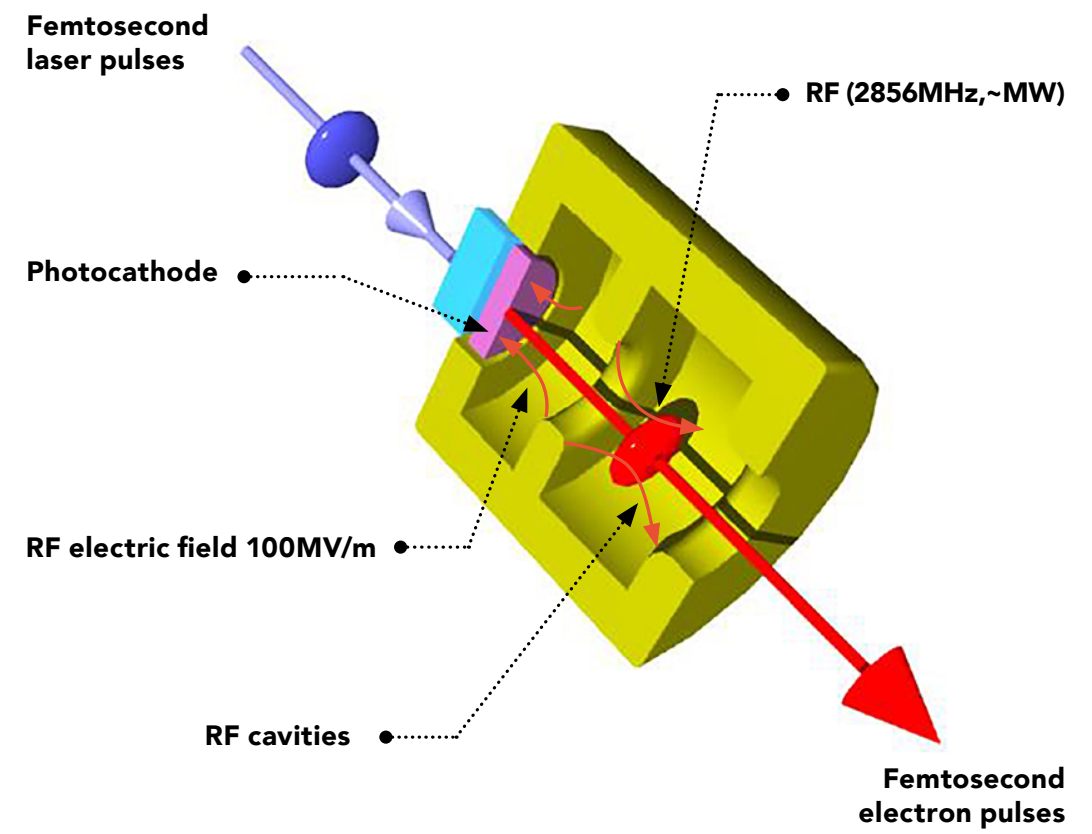

Through this ability to obtain diffraction patterns on such short timescales, the team was finally able to directly study the ways in which ultrafast physical processes unfold. from solids to liquids or from liquids to solids. In addition, it would provide unprecedented opportunities to view the dynamics of individual molecules

\section{ELECTRON DIFFRACTION PATTERNS}

structures aren't the only desirable outcome of UEM. Perhaps even more fascinating physical properties can be gathered thanks to the quantum nature of electrons themselves. Since electrons are quantum particles, they can be described as both particles and waves. a small gap, they will pass through

Concept of UEM with relativistic femtosecond electron pulses
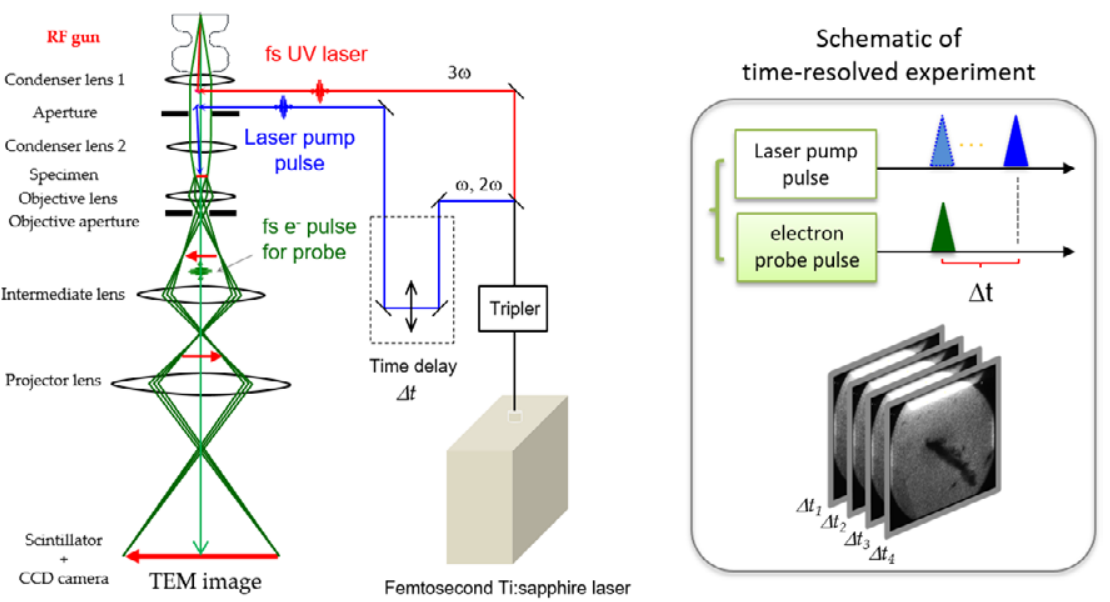

macroscopic wave When measung crystalline sample, the elastically scattered electrons contribute in typical diffraction patterns. The diffraction patterns are used to obtain quantitative data including atomic structures, orientation relationship, and crysta defects in materials.

Thanks to this effect, Dr Yang's team was able to use UEM to directly measure the structural dynamics of crystalline materials. "In our ultrafast electron diffraction (UED) study, we succeeded in the acquisition of highquality high-resolution diffraction patterns with both single shot and time-resolved experiments, with of $180 \mathrm{fs}$, " Dr Yang describes.

Through this ability to obtain diffraction patterns on such short we to directy stum was finally which ultrafast physical processes unfold. "This technique has been used successfully to observe the ultrafast dynamics of various photoinduced phase transitions in materials, including the photo-excited melting dynamics in a single crystal gold nanofilm," continues Dr Yang. "This was obtained by the single-shot UED measurement with a $3 \mathrm{MeV}$ and $90 \mathrm{fs}$ electron pulse." With the success of these diffraction experiments, DrYang's team has made significant progress towards achieving the

\section{ADVANCING MATERIALS PHYSICS} Their innovative redesign of the and his colleagues to demonstrate for the first time that nanoscale imaging need not be limited by the shortcomings of time-resolved TEM. By accelerating electrons to relativistic speeds, both diffraction pattern and direct images of samples can be obtained on atomic spatial scales, and on almost unimaginably short timescales. With further research, the technique of relativistic UEM could become a staple of materials physics future to make reliach future to make reliable measurements of highly intricate biological

\section{Behind the Research}

Dr Jinfeng Yang

E: yang@sanken.osaka-u.ac.jp T: +81668794285

W: https://www.sanken.osaka-u.ac.jp/en/organization/nnc/nnc_02/

W: https://researchmap.jp/read0134206

Research Objectives

Dr Jinfeng Yang has dedicated his work to the

\section{Detail}

\section{Jinfeng Yang}

Associate professor

The Institute of Scientific and Industrial Research (ISIR) Osaka University

\section{Bio} applications of high-brightness femtosecond/ picosecond pulsed electron beams in the particle accelerator field over the twenty years. His curre ultrafast electron diffraction/microscopy with relativistic femtosecond electron pulses and the study of ultrafast phenomena including structura dynamics and chemical/biochemical reactions.

\section{Funding}

Nherks were supported by JSPS KAKENHI Grant Grant-in-Aid for Scientific Research (A) and JP16K13687 of Challenging Research Exploratory, Japan.

\section{Collaborators}

Prof Yoichi Yoshida and Drs Koichi Kan and Masao Gohdo of the Institute of Scientific and Industrial femtosecond electron pulse generation. - Profs Katsumi Tanimura, Hidehiro Yasuda of the Research Center for Ultra-High Voltage Electron Microscopy (UHVEM) in Osaka University for the development of ultrafast electron diffraction/microscopy.

Drs Toshikatu Takatomi, Taro Konomi, Profs. Takayuki Saeki, Nobuhio Tenni Ma, Junji Urakawa of the High the development of the high-quality RF gun.

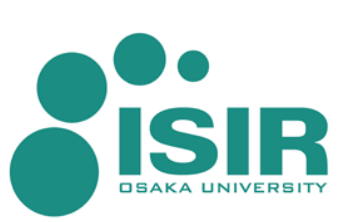

\section{References}

Yang, J., Yoshida, Y. and Yasuda, H. (2018). Ultrafast electron microscopy with relativistic femtosecond electron pulses. Microscopy, 67(5), p.291-295.

Yang, J. and Yoshida, Y. (2019). Relativistic Ultrafast Electron Microscopy: Single-Shot Diffraction Imaging wit Matter Physics.

Giret, Y., Naruse, N., Daraszewicz, S.L., Murooka, Y., Yang, J., Duffy, D.M., Shluger, A.L. and Tanimura, K. (2013). Determination of transient atomic structure of laser-excited Deterict

Yang, J., Yoshida, Y. and Shibata, H. (2015). Femtosecond Communications in Japan, 98(11), p.50-57.

Murooka, Y., Naruse, N., Sakakihara, S., Ishimaru, M., Yang ectron pulses. Applied Physics Letters, 98

Yang, J., Gen, K., Naruse, N., Sakakihara, S., and Yoshida Y. (2020). A compact ultrafast electron diffractometer with Science, 4(4).

\section{Personal Response}

\section{What are you most proud of in your work?}

II I have worked on the generation and beam physics on high-brightness electron beams in the have developed various RF guns for the purs. I electron beam generation from femtosecond to picosecond. I succeeded in constructing an ultrafast pulse radiolysis instrument with $240 \mathrm{ts}$ temporal resolution in 2009 and first opened the study of radiation chemical processes in femtosecond timescales. Since 2013 , have been designing and
constructing the world's first prototype of UEM with relativistic femtosecond electron pulses. The current research is concerned with the study of ultrafast phenomena including structural dynamics, chemical
and biochemical reactions. 